\title{
Prevalence and morphological characterization of Aspergillus isolates of maize ear rot in Punjab
}

\author{
Harpreet Singh ${ }^{1}$, Harleen Kaur ${ }^{2}$ and Mandeep Singh Hunjan ${ }^{1}$ \\ ${ }^{1}$ Department of Plant Pathology, Punjab Agricultural University, Ludhiana-141 004, INDIA \\ ${ }^{2}$ Department of Plant Breeding and Genetics, Punjab Agricultural University, Ludhiana-141 004, INDIA \\ *Corresponding author. E-mail: harleenkaur@pau.edu
}

Received: October 30, 2015; Revised received: April 08, 2016; Accepted: July 12, 2016

\begin{abstract}
Among six fungal species viz.Aspergillus spp., Fusarium spp., Penicillum spp., Rhizopus spp., Trichoderma spp. and Alternaria spp. isolated from maize ear rot samples collected from five different districts of Punjab, the incidence of $A$. flavus was highest (42.15\%) followed by Penicillium spp. (20.75\%). The maximum frequency of A. flavus (57.10\%) was found in Hoshiarpur district, closely followed by Jalandhar. In all, thirty nine isolates of $A s-$ pergillus spp. (33 isolates of $A$. flavus and 6 isolates of $A$. niger) were characterized morphologically. Twenty isolates of $A$. flavus producing sclerotia were of L-type strains having sclerotia diameter $>400 \mu \mathrm{m}$. Isolates of $A$. flavus produced yellowish green, dark green and light green colonies and isolates of $A$. niger produced dark black colonies. Sterigmata in all the isolates of Aspergillus spp. were of uniseriate type. Based on colony diameter and growth rate per day all the 39 isolates of Aspergillus spp. were grouped into fast, medium and slow growing categories. Based on multivariate cluster analysis, the isolates of $A$. flavus were grouped into three distinct clusters each having 13,17 and 3 isolates respectively. In the present study, Aspergillus flavus was found predominantly associated species with the maize ear rot. Further, the morphological variation observed within Aspergillus flavus and $A$. niger indicated the need for proper surveillance and monitoring exclusively for the prevention of moulds in maize produce in Punjab before it reaches the consumer.
\end{abstract}

Keywords: Aspergillus flavus, A. niger, Ear rot, Isolate,Maize

\section{INTRODUCTION}

Ear rot is the most potentially damaging disease of maize crop (Gxasheka et al., 2015). Several fungal species viz: Aspergillus, Fusarium and Penicillum are associated with the maize grains in field as well as in storage. Ear rot disease results in reduced grain but the main loss from ear rot disease is due to the contamination of grain yield with mycotoxins which are a threat to safety of both humans and livestock (Bello et al., 2012). The direct economic impact of fungal and mycotoxin contamination in maize grains results mainly from a reduction in marketable volume, loss in value in the national markets, inadmissibility or rejection of products by the international market, and losses incurred from livestock disease, consequential morbidity and mortality. The average economic loss due to mycotoxin contamination is estimated at approximately one billion dollars, with aflatoxins representing a large proportion of this loss in the United States (Amaike and Keller, 2011). Aspergillus ear rot of maize is predominately caused by A. flavus and is prevalent where drought conditions occurs and can lead to the accumulation of aflatoxins in grains (Nicholson et al., 2004). Aflatoxicosis causes acute liver damage, liver cirrho- sis, induction of tumors, impaired central nervous system, skin disorder and hormonal defects.

Morphological characterization is the primary tool for the identification of Aspergillus species. Although molecular methods continue to improve and become more rapidly available, microscopy and culture remain commonly used and essential tools for identification of Aspergillus spp. (McClenny, 2005). As with fungi in general, Aspergillus taxonomy is complex and ever evolving. The genus is easily identified by its characteristic conidiophore, but species identification and differentiation is complex, for it is traditionally based on a range of morphological features. Macromorphological features which are often considered include conidial and mycelial color, colony diameter, colony pigmentation, production of exudates and soluble pigments, presence of sclerotia and cleistothecia. Micromorphology characterization is mainly dependent on seriation, shape and size of vesicle, conidia and stipe morphology and morphology of cleistothecia and ascospores (Klich, 2002). The best quality foods produced in some nations are rejected for export because of aflatoxin levels exceeding the tolerable limit, resulting in millions of dollars in losses. Its major cause is the presence of moulds especially Aspergillus flavus asso- 
ciated with grains at the time of harvesting or storage. Since crop and aflatoxins are of paramount importance to Punjab State and no studies have been conducted on presence of different fungal genera associated with maize ear rot in Punjab. Keeping this in mind, the present study was focused on prevalence of different fungal genera associated with maize ear rot in different maize growing areas of Punjab and to characterize species of Aspergillus morphologically.

\section{MATERIALS AND METHODS}

Sampling and isolations: Maize ear rot samples were collected from different maize growing districts of Punjab viz., Ludhiana, Jalandhar, Hoshiarpur, Gurdaspur and Kapurthala during Kharif 2013 and spring 2014. Isolations of fungal spp. associated with maize ear rot were made from the infected samples on potato dextrose agar (PDA) medium. Six fungal spp. Aspergillus spp., Fusarium spp., Penicillum spp., Rhizopus spp., Trichoderma spp. and Alternaria spp. were isolated from the diseased samples. The cultures thus obtained were purified and maintained on PDA slants for further studies.

Morphological characteristics of Aspergillus isolates: Thirty three isolates of Aspergillus flavus and six isolates of $A$. niger were characterized morphologically. A $5 \mathrm{~mm}$ mycelium disc was cut from the actively growing edge of 5 day old culture and placed on Petri dish containing $20 \mathrm{ml}$ PDA and incubated at $25 \pm 2^{\circ} \mathrm{C}$. Colony color, pigmentation (Anonymous, 2015), type of sterigmata, growth rate and production of sclerotia were observed after 7 days of incubation. Observations on radial growth pattern were taken at every $24 \mathrm{hrs}$ interval. Each isolate was replicated thrice. The diameter of conidia, size of conidiophore and sclerotia diameter were observed microscopically and measurements were taken using image analysis software connected to Lecia DM 3000 microscope.

Multivariate cluster analysis was done for both qualitative and quantitative cultural characters using the Unweighted Pair Group Mean Average (UPGMA) with statistical analysis tool PAST ver. 2.1.5 and dendrogram was constructed.

\section{RESULTS}

Prevalence of ear rot fungi: The data on frequency of occurrence of different microorganisms associated with maize ear rot in different districts of Punjab are presented in Table 1. The data indicated that maximum incidence of A. flavus (57.1\%) and Fusarium spp. (14.8\%) was found in Hoshiarpur district, closely followed by Jalandhar (54.5\%). All six fungal species (Aspergillus spp., Fusarium spp., Penicillum spp., Rhizopus spp., Trichoderma spp. and Alternaria spp.) were isolated from Ludhiana samples. The maximum incidence of A. niger was observed in Ludhiana (9.9\%) district while minimum in Hoshiarpur $(7.1 \%)$ district.
The frequency of Penicillum spp. (33.7\%) and Rhizopus spp. (28.1\%) was observed maximum in Gurdaspur and minimum in Hoshiarpur district.

Among all the 7 species (A. flavus, A. niger, Fusarium spp., Penicillum spp., Rhizopus spp., Trichoderma spp. and Alternaria spp.) associated with maize ear rot, the incidence of A. flavus (42.15\%) was highest followed by Penicillium spp. (20.75\%) and Rhizopus spp. (15.67\%). The incidence of Alternaria spp. was lowest only up to 0.67 per cent (Fig. 1).

\section{Morphological characterization}

Qualitative characters: The isolates of A. flavus within themselves do not show much variation in colony color. Except Af 12, Af 13, Af 16, Af 19 and Af 30 , rest all isolates were having yellow green colonies (Table 2). Out of these, four isolates Af 12, Af 13, Af 16 and Af 19 were having dark green colonies and one isolate Af 30 was having light green colonies. The pigmentation of all the isolates varied from creamish to creamish yellow. All isolates of A. niger were having dark black colonies with cream color pigmentation whereas An 34 had dark black colonies with whitish margin and yellow color pigmentation.

Based on the average colony growth, all the isolates of A. flavus and A. niger were categorized as fast (> 75 $\mathrm{cm})$, medium $(75-80 \mathrm{~cm})$ and slow $(<80 \mathrm{~cm})$ growing (Table 3). The data indicated that eight isolates (Af 2, Af 5, Af 9, Af 13, Af 18, Af 27, Af 30 and Af 33) of $A$. flavus were slow growing with average colony diameter of $<75 \mathrm{~mm}$ and average growth rate per day ranging from 9.3 to $10.6 \mathrm{~mm}$. Six isolates (Af 1, Af 4, Af 11, Af 15, Af 31 and Af 32) had medium growth with average colony diameter in the range of 75-80 $\mathrm{mm}$ and average colony growth rate ranging from 10.7 to 10.9 $\mathrm{mm}$ per day. Rest of the isolates were relatively fast growing with average colony diameter of $>80 \mathrm{~mm}$ and average colony growth rate of 11.0 to $12.7 \mathrm{~mm}$ per day. Similarly, in isolates of A. niger, three isolates (An 34, An 38 and An 39) were slow growing with average colony diameter of $<75 \mathrm{~mm}$ and average

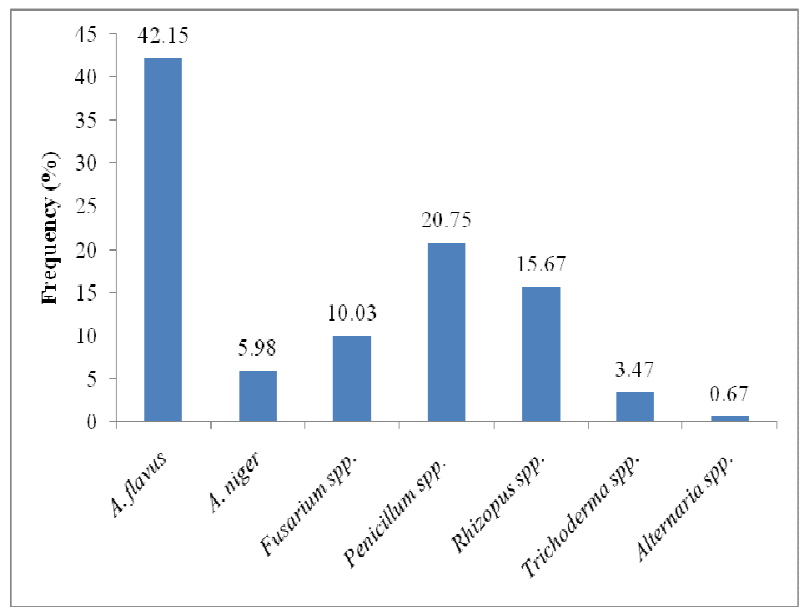

Fig. 1. Prevalence of different microorganisms associated with ear rot of maize. 
Harpreet Singh et al. / J. Appl. \& Nat. Sci. 8 (3): 1229 - 1234 (2016)

Table 1. Frequency of occurrence of different microorganisms associated with maize ear rot in different districts of Punjab.

\begin{tabular}{lccccc}
\hline \multirow{2}{*}{ Microorganism } & \multicolumn{5}{c}{ Frequency (\%) } \\
\cline { 2 - 6 } & Ludhiana & Jalandhar & Hoshiarpur & Gurdaspur & Kapurthala \\
\hline A. flavus & 31.3 & 54.5 & 57.1 & 30.6 & 45.5 \\
A. niger & 9.9 & 0 & 7.1 & 0 & 9.1 \\
Fusarium spp. & 13.9 & 9.1 & 14.8 & 0 & 8.1 \\
Penicillum spp. & 15.7 & 18.2 & 14.3 & 33.7 & 27.3 \\
Rhizopus spp. & 17.7 & 18.7 & 0 & 28.1 & 0 \\
Trichoderma spp. & 5.9 & 9.1 & 0 & 0 & 0 \\
Alternaria spp. & 4 & 0 & 0 & 0 \\
\hline
\end{tabular}

growth rate ranging from 5.5 to $9.5 \mathrm{~mm}$ per day. Only one isolate An 35 had medium growth with average colony diameter in the range of 75-80 $\mathrm{mm}$ and average colony growth rate of 9.6 to $10.9 \mathrm{~mm}$ per day. Fast growing isolates, An 36 and An 37 have average colony diameter of $>80 \mathrm{~mm}$ and average colony growth rate of 11.0 to $11.6 \mathrm{~mm}$ per day.

Quantitative characters: All the isolates of A. flavus and $A$. niger produced abundant, single celled and round conidia while conidiophores have vesicles bearing conidia on uniseriate sterigmata. The data revealed that conidia diameter of A. flavus isolate Af 6 was maximum (30.7), whereas it was minimum (19.8) in Af 12 isolate (Table 2). The size of conidiophores of $A$. flavus isolates varied greatly in length and slightly in width. The mean length of conidiophores of A. flavus isolates varied from 227.5 to $1204.2 \mu \mathrm{m}$ and mean width varied from 11.2 to $28.9 \mu \mathrm{m}$. Mean conidia diameter of A. niger isolates was in the range of 26.3 to $30.7 \mu \mathrm{m}$, found minimum in An 38 and maximum in An 39 isolate. The mean length and width of conidiophores of $A$. niger isolates was in the range of 512.1 to $824.7 \mu \mathrm{m}$ and 8.7 to $19.7 \mu \mathrm{m}$ respectively. The isolate An 36 had minimum width and isolate An 39 had maximum width.
Out of 39 isolates, only 20 isolates of A. flavus produced sclerotia. Size of sclerotia varies from 747.3 to 1155.2 $\mu \mathrm{m}$, found maximum and minimum in isolate Af 11 and Af 22, respectively. All the isolates producing sclerotia were of L-type having sclerotia diameter of $>400 \mu \mathrm{m}$.

Cluster analysis: Based on morphological characters, isolates of A. flavus were grouped into three clusters each having 13, 17 and 3 isolates respectively (Fig. 2). One isolate Af 33 from Kapurthala district out group cluster I with minimum growth rate per day $(9.5 \mathrm{~mm})$ and least conidiophores length $(227.50 \mu \mathrm{m})$. Cluster-II containing 17 isolates can be differentiated from the other two clusters on the basis of conidia size and growth rate (Table 4$)$. The mean conidial diameter was minimum $(28.2 \mu \mathrm{m})$ and mean growth rate per day was found maximum (11.2) in cluster-II than other clusters. However size of conidiophores was more than cluster-I but less than cluster-III. Sclerotia diameter was also minimum (936.5) in cluster-II than cluster-III. ClusterI can be differentiated from the other two clusters on the basis of sclerotia production. There was no sclerotia production in isolates grouped in cluster-I. Cluster-III containing 3 isolates had largest size of conidia $(29.7 \mu \mathrm{m})$, conidiophore $(1059.1 \mu \mathrm{m})$ and sclerotia

m

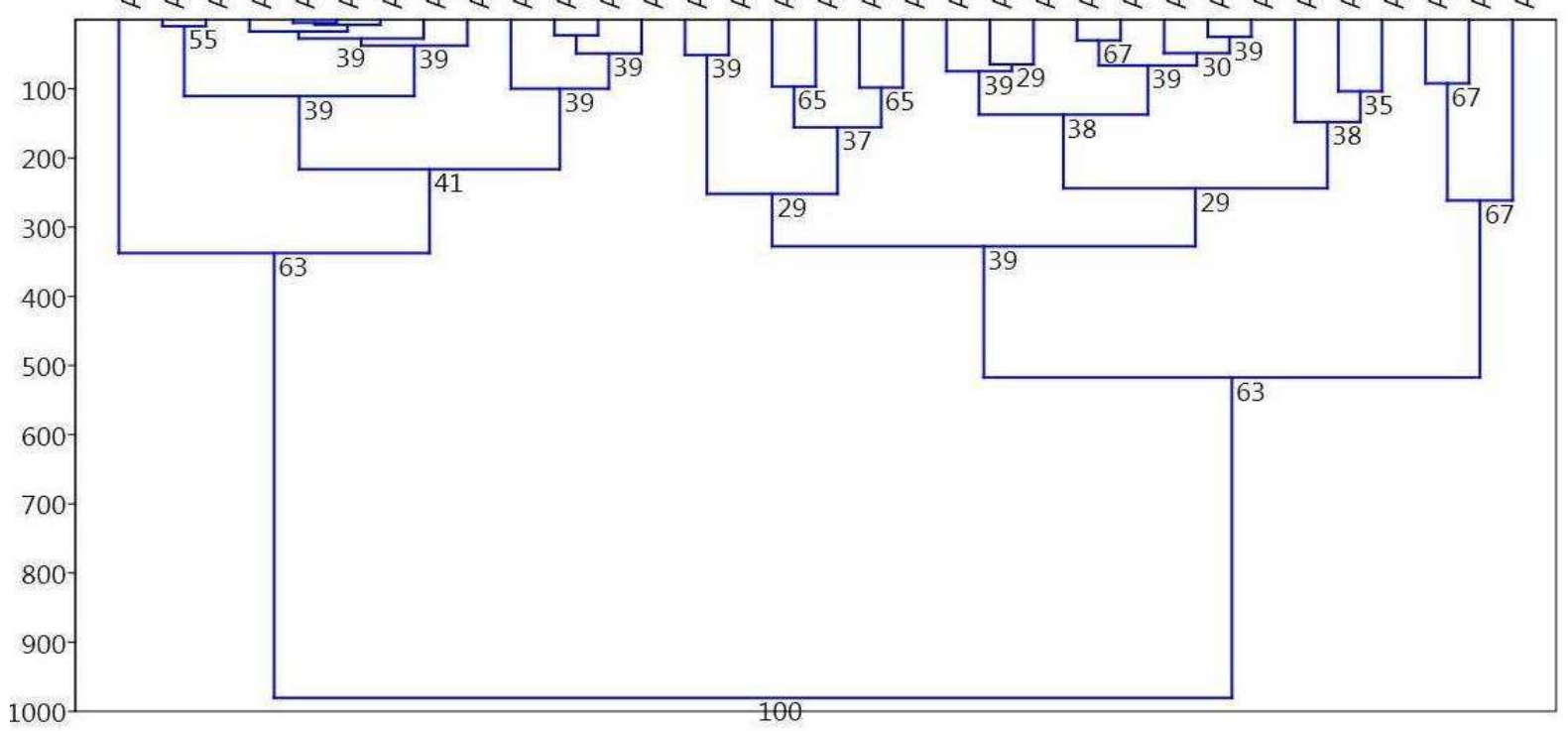

Fig. 2. Dendrogram showing clustering of A. flavus isolates based on morphological characteristics. 
Harpreet Singh et al. / J. Appl. \& Nat. Sci. 8 (3): 1229 - 1234 (2016)

Table 2. Morphological characteristics of isolates of Aspergillus spp. collected from different maize growing districts of Punjab.

\begin{tabular}{|c|c|c|c|c|c|c|}
\hline Isolate & Colony color & Pigmentation & $\begin{array}{l}\text { Diameter of conidia } \\
(\mu \mathrm{m})^{*}\end{array}$ & $\begin{array}{l}\text { Conidiophore size } \\
(\mu \mathrm{m})^{*}\end{array}$ & & $\begin{array}{ll}\begin{array}{l}\text { Diameter } \\
\text { sclerotia }\end{array} \\
\end{array}$ \\
\hline & & & & Length & Width & $(\mu \mathrm{m})^{*}$ at $5 X$ \\
\hline Af 1 & Yellow green & Creamish & $\begin{array}{l}30.0 \\
(26.3-34.6)\end{array}$ & $\begin{array}{l}952.7 \\
(855.7-1042.4)\end{array}$ & $\begin{array}{l}17.6 \\
(15.0-19.2)\end{array}$ & $\begin{array}{l}1027.6 \\
(913.2-1277.7)\end{array}$ \\
\hline Af 2 & Yellow green & Creamish & $\begin{array}{l}28.3 \\
(23.9-38.4)\end{array}$ & $\begin{array}{l}588.3 \\
(558.8-636.2)\end{array}$ & $\begin{array}{l}23.1 \\
(20.3-27.0)\end{array}$ & $\begin{array}{l}898.9 \\
(820.4-957.4)\end{array}$ \\
\hline Af 3 & Yellow green & Creamish yellow & $\begin{array}{l}26.9 \\
(23.4-31.3)\end{array}$ & $\begin{array}{l}580.1 \\
(544.8-615.5)\end{array}$ & $\begin{array}{l}22.3 \\
(18.1-24.5)\end{array}$ & $\begin{array}{l}861.3 \\
(806.6-912.0)\end{array}$ \\
\hline Af 4 & Yellow green & Creamish & $\begin{array}{l}29.9 \\
(21.9-38.1)\end{array}$ & $\begin{array}{l}1020.5 \\
(920.7-1082.8)\end{array}$ & $\begin{array}{l}19.0 \\
(15.2-24.8)\end{array}$ & $\begin{array}{l}965.0 \\
(915.3-1036.1)\end{array}$ \\
\hline Af 5 & Yellow green & Creamish & $\begin{array}{l}29.0 \\
(25.6-35.4)\end{array}$ & $\begin{array}{l}1204.2 \\
(1200.2-1208.2)\end{array}$ & $\begin{array}{l}21.2 \\
(19.2-23.5)\end{array}$ & $\begin{array}{l}851.7 \\
(775.7-938.8)\end{array}$ \\
\hline Af 6 & Yellow green & Creamish & $\begin{array}{l}30.7 \\
(27.2-33.0)\end{array}$ & $\begin{array}{l}577.9 \\
(566.5-589.4)\end{array}$ & $\begin{array}{l}11.2 \\
(8.5-14.4)\end{array}$ & - \\
\hline Af 7 & Yellow green & Creamish yellow & $\begin{array}{l}24.5 \\
(16.8-28.9)\end{array}$ & $\begin{array}{l}572.3 \\
(506.6-607.3)\end{array}$ & $\begin{array}{l}15.8 \\
(11.9-19.2)\end{array}$ & - \\
\hline Af 8 & Yellow green & Creamish & $\begin{array}{l}27.5 \\
(20.4-32.4)\end{array}$ & $\begin{array}{l}453.7 \\
(433.3-474.2)\end{array}$ & $\begin{array}{l}17.1 \\
(14.4-21.5)\end{array}$ & - \\
\hline Af 9 & Yellow green & Creamish & $\begin{array}{l}30.4 \\
(22.5-34.1)\end{array}$ & $\begin{array}{l}428.5 \\
(402.6-489.5)\end{array}$ & $\begin{array}{l}15.6 \\
(12.1-18.1)\end{array}$ & $\begin{array}{l}1068.8 \\
(1028.6-1121.6)\end{array}$ \\
\hline Af 10 & Yellow green & Creamish & $\begin{array}{l}28.7 \\
(23.1-38.9)\end{array}$ & $\begin{array}{l}783.2 \\
(780.5-785.9)\end{array}$ & $\begin{array}{l}14.0 \\
(12.1-16.8)\end{array}$ & - \\
\hline Af 11 & Yellow green & Creamish & $\begin{array}{l}30.2 \\
(26.3-33.4)\end{array}$ & $\begin{array}{l}278.2 \\
(217.4-339.4)\end{array}$ & $\begin{array}{l}15.6 \\
(12.1-19.6)\end{array}$ & $\begin{array}{l}747.3 \\
(708.6-807.3)\end{array}$ \\
\hline Af 12 & $\begin{array}{l}\text { Dark green with } \\
\text { whitish periphery }\end{array}$ & Creamish & $\begin{array}{l}19.8 \\
(12.8-32.3)\end{array}$ & $\begin{array}{l}727.4 \\
(696.1-756.5)\end{array}$ & $\begin{array}{l}14.6 \\
(12.1-17.3)\end{array}$ & $\begin{array}{l}855.1 \\
(778.7-971.6)\end{array}$ \\
\hline Af 13 & $\begin{array}{l}\text { Dark green with } \\
\text { whitish periphery }\end{array}$ & Creamish & $\begin{array}{l}20.9 \\
(23.4-31.3)\end{array}$ & $\begin{array}{l}677.8 \\
(610.7-717.6)\end{array}$ & $\begin{array}{l}18.9 \\
(15.2-22.4)\end{array}$ & - \\
\hline Af 14 & Yellow green & Creamish & $\begin{array}{l}26.9 \\
(13.8-33.3)\end{array}$ & $\begin{array}{l}709.3 \\
(685.6-762.2)\end{array}$ & $\begin{array}{l}15.3 \\
(11.9-18.8)\end{array}$ & $\begin{array}{l}924.9 \\
(788.1-1014.6)\end{array}$ \\
\hline Af 15 & Yellow green & Creamish & $\begin{array}{l}24.0 \\
(14.3-35.3)\end{array}$ & $\begin{array}{l}644.6 \\
(598.6-667.8)\end{array}$ & $\begin{array}{l}22.1 \\
(18.1-26.6)\end{array}$ & $\begin{array}{l}914.8 \\
(771.8-1041.9)\end{array}$ \\
\hline Af 16 & $\begin{array}{l}\text { Dark green with } \\
\text { white periphery }\end{array}$ & Creamish & $\begin{array}{l}30.3 \\
(21.9-40.6)\end{array}$ & $\begin{array}{l}715.9 \\
(677.0-750.2)\end{array}$ & $\begin{array}{l}16.2 \\
(14.2-19.6)\end{array}$ & - \\
\hline Af 17 & Yellow green & Creamish & $\begin{array}{l}28.7 \\
(22.9-36.9)\end{array}$ & $\begin{array}{l}774.1 \\
(757.1-787.4)\end{array}$ & $\begin{array}{l}16.5 \\
(12.8-19.2)\end{array}$ & $\begin{array}{l}1118.8 \\
(1071.8-1171.6)\end{array}$ \\
\hline Af 18 & Yellow green & Creamish & $\begin{array}{l}29.0 \\
(22.2-38.4)\end{array}$ & $\begin{array}{l}657.3 \\
(606.7-725.7)\end{array}$ & $\begin{array}{l}14.1 \\
(12.8-16.6)\end{array}$ & - \\
\hline Af 19 & $\begin{array}{l}\text { Dark green with } \\
\text { white periphery }\end{array}$ & Creamish & $\begin{array}{l}30.4 \\
(24.8-35.7)\end{array}$ & $\begin{array}{l}471.7 \\
(460.1-490.9)\end{array}$ & $\begin{array}{l}12.4 \\
(10.1-15.9)\end{array}$ & - \\
\hline Af 20 & Yellow green & Creamish & $\begin{array}{l}29.0 \\
(25.4-33.1)\end{array}$ & $\begin{array}{l}637.8 \\
(618.8-656.8)\end{array}$ & $\begin{array}{l}22.3 \\
(18.1-28.6)\end{array}$ & $\begin{array}{l}886.0 \\
(857.4-936.4)\end{array}$ \\
\hline Af 21 & Yellow green & Creamish & $\begin{array}{l}30.0 \\
(23.8-35.8)\end{array}$ & $\begin{array}{l}574.6 \\
(568.5-580.7)\end{array}$ & $\begin{array}{l}23.1 \\
(21.3-25.6)\end{array}$ & $\begin{array}{l}919.6 \\
(915.7-923.1)\end{array}$ \\
\hline Af 22 & Yellow green & Creamish & $\begin{array}{l}29.35 \\
(26.3-37.3)\end{array}$ & $\begin{array}{l}594.7 \\
(590.5-598.9)\end{array}$ & $\begin{array}{l}19.7 \\
(16.8-23.3)\end{array}$ & $\begin{array}{l}1155.2 \\
(1125.0-1225.0)\end{array}$ \\
\hline Af 23 & Yellow green & Creamish & $\begin{array}{l}30.6 \\
(23.8-36.0)\end{array}$ & $\begin{array}{l}667.45 \\
(644.4-690.5)\end{array}$ & $\begin{array}{l}20.4 \\
(18.6-22.4)\end{array}$ & $\begin{array}{l}1081.4 \\
(982.3-1143.2)\end{array}$ \\
\hline Af 24 & Yellow green & Creamish & $\begin{array}{l}29.8 \\
(20.3-36.0)\end{array}$ & $\begin{array}{l}276.23 \\
(232.2-301.4)\end{array}$ & $\begin{array}{l}14.3 \\
(12.1-16.8)\end{array}$ & $\begin{array}{l}843.9 \\
(797.4-893.0)\end{array}$ \\
\hline Af 25 & Yellow green & Creamish & $\begin{array}{l}29.0 \\
(23.6-34.6)\end{array}$ & $\begin{array}{l}473.4 \\
(419.1-504.6)\end{array}$ & $\begin{array}{l}16.1 \\
(12.8-21.4)\end{array}$ & - \\
\hline Af 26 & Yellow green & Creamish & $\begin{array}{l}29.6 \\
(26.3-34.1)\end{array}$ & $\begin{array}{l}414.1 \\
(349.2-449.8)\end{array}$ & $\begin{array}{l}13.4 \\
(9.8-17.4)\end{array}$ & $\begin{array}{l}802.2 \\
(633.5-893.7)\end{array}$ \\
\hline Af 27 & Yellow green & Creamish & $\begin{array}{l}28.8 \\
(24.6-36.4)\end{array}$ & $\begin{array}{l}455.7 \\
(355.4-562.5)\end{array}$ & $\begin{array}{l}13.0 \\
(10.1-15.2)\end{array}$ & $\begin{array}{l}1025.6 \\
(1021.7-1030.6)\end{array}$ \\
\hline Af 28 & Yellow green & Creamish & $\begin{array}{l}30.5 \\
(23.4-41.5)\end{array}$ & $\begin{array}{l}485.6 \\
(403.8-590.5)\end{array}$ & $\begin{array}{l}12.7 \\
(7.5-16.8)\end{array}$ & - \\
\hline Af 29 & Yellow Green & Creamish & $\begin{array}{l}26.2 \\
(23.4-34.6)\end{array}$ & $\begin{array}{l}773.0 \\
(710.4-896.0)\end{array}$ & $\begin{array}{l}21.8 \\
(18.1-26.6)\end{array}$ & $\begin{array}{l}916.2 \\
(850.1-986.1)\end{array}$ \\
\hline Af 30 & Light green & Creamish & $\begin{array}{l}29.2 \\
(24.2-37.2)\end{array}$ & $\begin{array}{l}491.5 \\
(434.9-554.0)\end{array}$ & $\begin{array}{l}23.5 \\
(19.0-27.8)\end{array}$ & - \\
\hline Af 31 & Yellow green & Creamish & $\begin{array}{l}29.9 \\
(24.5-39.2)\end{array}$ & $\begin{array}{l}400.9 \\
(693.8-461.4)\end{array}$ & $\begin{array}{l}17.8 \\
(14.4-19.6)\end{array}$ & $\begin{array}{l}899.3 \\
(811.0-983.8)\end{array}$ \\
\hline Af 32 & Yellow green & Creamish & $\begin{array}{l}29.7 \\
(23.4-36.0)\end{array}$ & $\begin{array}{l}433.7 \\
(418.0-446.7)\end{array}$ & $\begin{array}{l}15.6 \\
(13.8-19.6)\end{array}$ & - \\
\hline Af 33 & Yellow green & Creamish & $\begin{array}{l}29.1 \\
(25.3-35.4)\end{array}$ & $\begin{array}{l}227.5 \\
(367.9-560.1)\end{array}$ & $\begin{array}{l}28.9 \\
(22.5-33.4)\end{array}$ & - \\
\hline An 34 & $\begin{array}{l}\text { Dark black with } \\
\text { white margin }\end{array}$ & Yellowish & $\begin{array}{l}27.6 \\
(23.0-31.7)\end{array}$ & $\begin{array}{l}744.3 \\
(700.9-787.6)\end{array}$ & $\begin{array}{l}11.0 \\
(9.8-12.9)\end{array}$ & - \\
\hline An 35 & Dark black & Creamish & $\begin{array}{l}29.6 \\
(23.9-35.8)\end{array}$ & $\begin{array}{l}695.3 \\
(612.6-778.1)\end{array}$ & $\begin{array}{l}8.8 \\
(6.7-12.9)\end{array}$ & - \\
\hline An 36 & Dark black & Creamish & $\begin{array}{l}30.6 \\
(23.9-38.1)\end{array}$ & $\begin{array}{l}512.1 \\
(478.7-545.5)\end{array}$ & $\begin{array}{l}8.7 \\
(7.7-9.7)\end{array}$ & - \\
\hline An 37 & Dark black & Creamish & $\begin{array}{l}27.2 \\
(22.0-31.0)\end{array}$ & $\begin{array}{l}716.5 \\
(688.1-742.6)\end{array}$ & $\begin{array}{l}9.3 \\
(8.0-11.9)\end{array}$ & - \\
\hline An 38 & Dark black & Creamish yellow & $\begin{array}{l}26.3 \\
(21.3-36.9)\end{array}$ & $\begin{array}{l}824.7 \\
(805.1-844.3)\end{array}$ & $\begin{array}{l}9.6 \\
(6.7-12.8)\end{array}$ & - \\
\hline An 39 & Dark black & Creamish & $\begin{array}{l}30.7 \\
(25.8-36.3)\end{array}$ & $\begin{array}{l}695.3 \\
(678.1-712.6)\end{array}$ & $\begin{array}{l}19.7 \\
(14.2-22.5)\end{array}$ & - \\
\hline
\end{tabular}


Table 3. Categorization of isolates of Aspergillus spp. on the basis of their colony growth.

\begin{tabular}{|c|c|c|c|c|c|c|c|}
\hline \multicolumn{2}{|c|}{ Aspergillus spp. } & \multicolumn{4}{|l|}{ Isolate (s) } & $\begin{array}{l}\text { Colony diameter } \\
(\mathbf{m m})\end{array}$ & $\begin{array}{l}\text { Growth rate/ } \\
\text { day }(\mathbf{m m})\end{array}$ \\
\hline \multirow{3}{*}{\multicolumn{2}{|c|}{ A. flavus }} & \multirow{3}{*}{\multicolumn{4}{|c|}{$\begin{array}{l}\text { Af } 2, \text { Af } 5, \text { Af } 9, \text { Af } 13, \text { Af } 18, \text { Af } 27, \text { Af } 30, \text { Af } 33 \\
\text { Af } 1, \text { Af } 4, \text { Af } 11, \text { Af } 15, \text { Af } 31, \text { Af } 32 \\
\text { Af } 3, \text { Af } 6, \text { Af } 7, \text { Af } 8, \text { Af } 10, \text { Af } 12, \text { Af } 14, \text { Af } 16, \text { Af } 17, \text { Af } \\
19, \text { Af } 20, \text { Af } 21, \text { Af } 22, \text { Af } 23, \text { Af } 24, \text { Af } 25, \text { Af } 26, \text { Af } 28, \\
\text { Af } 29\end{array}$}} & $<75$ & $9.3-10.6$ \\
\hline & & & & & & $75-80$ & $10.7-10.9$ \\
\hline & & & & & & $>80$ & $11.0-12.7$ \\
\hline \multirow{3}{*}{\multicolumn{2}{|c|}{ A. niger }} & \multirow{3}{*}{\multicolumn{4}{|c|}{$\begin{array}{l}\text { An } 34, \text { An } 38, \text { An } 39 \\
\text { An } 35 \\
\text { An } 36 \text {, An } 37 \\
\end{array}$}} & \multirow{3}{*}{$\begin{array}{l}<75 \\
75-80 \\
>80\end{array}$} & $5.5-9.5$ \\
\hline & & & & & & & $9.6-10.9$ \\
\hline & & & & & & & $11.0-11.6$ \\
\hline \multirow{3}{*}{ Cluster } & \multirow{3}{*}{\multicolumn{2}{|c|}{ Isolates }} & \multirow{3}{*}{$\begin{array}{l}\text { Conidia } \\
\text { diameter } \\
(\mu \mathrm{m})^{*}\end{array}$} & \multirow{2}{*}{\multicolumn{2}{|c|}{$\begin{array}{c}\text { Size of conidiophore } \\
(\mu \mathrm{m})^{*}\end{array}$}} & \multirow{3}{*}{$\begin{array}{c}\text { Sclerotia } \\
\text { diameter } \\
(\mu \mathrm{m})^{*}\end{array}$} & \multirow{3}{*}{$\begin{array}{l}\text { Mean growth } \\
\text { rate per day } \\
(\mathbf{m m})^{*}\end{array}$} \\
\hline & & & & & & & \\
\hline & & & & Length & Width & & \\
\hline \multirow[t]{2}{*}{ I } & \multirow{2}{*}{\multicolumn{2}{|c|}{$\begin{array}{l}\text { Af } 33, \text { Af } 6, \text { Af } 7, \text { Af } 8, \text { Af } 19, \\
\text { Af } 25, \text { Af } 28, \text { Af } 30, \text { Af } 32, \text { Af } \\
10, \text { Af } 13, \text { Af } 18, \text { Af } 16\end{array}$}} & 28.5 & 540.1 & 16.7 & - & 11.1 \\
\hline & & & $(20.9-30.7)$ & $\begin{array}{l}(227.5- \\
787.2)\end{array}$ & $\begin{array}{l}(11.3- \\
28.9)\end{array}$ & & $(9.5-12.7)$ \\
\hline \multirow[t]{2}{*}{ II } & \multirow{2}{*}{\multicolumn{2}{|c|}{$\begin{array}{l}\text { Af } 9, \text { Af } 27, \text { Af } 11, \text { Af } 24, \text { Af } \\
26, \text { Af } 31, \text { Af } 12, \text { Af } 14, \text { Af } 29, \\
\text { Af } 15, \text { Af } 20, \text { Af } 3, \text { Af } 2, \text { Af } 21, \\
\text { Af } 17, \text { Af } 22, \text { Af } 23\end{array}$}} & 28.2 & 560.3 & 18.3 & 936.5 & 11.2 \\
\hline & & & $(19.9-30.6)$ & $\begin{array}{l}(276.2- \\
774.1)\end{array}$ & $\begin{array}{l}(13.1- \\
23.2)\end{array}$ & $(747.3-1155.2)$ & $(9.3-12.2)$ \\
\hline \multirow[t]{2}{*}{ III } & \multirow{2}{*}{\multicolumn{2}{|c|}{ Af 4, Af 1, Af 5}} & 29.7 & 1059.1 & 19.3 & 948.2 & 10.7 \\
\hline & & & $(29.0-30.0)$ & $\begin{array}{l}(952.7- \\
1204.0)\end{array}$ & $\begin{array}{l}(17.7- \\
21.3)\end{array}$ & $(851.8-1027.7)$ & $(10.6-10.8)$ \\
\hline
\end{tabular}

*Mean of 50 observations and range is given in parenthesis- Sclerotia absent

$(948.2 \mu \mathrm{m})$ than cluster-I and cluster-II isolates. However mean growth per day was minimum $(10.7 \mathrm{~mm} /$ day) in cluster-III than other two clusters. Isolate Af 5 (Ludhiana isolate) outgroup cluster-III with maximum conidiophore length of $1204.2 \mu \mathrm{m}$.

\section{DISCUSSION}

Ear rot fungi are associated with maize grains in the field as well as under storage conditions. Out of six fungal species isolated, A. flavus was found predominantly associated with maize grains. Its frequency was found maximum in Hoshiarpur district of Punjab. These findings support the observations made by several workers (Atehnkeng et al., 2008 and Abriba et al., 2013). Atehnkeng et al. (2008) also found that the incidence of Aspergillus species was highest on maize grains and within Aspergilllus, A. flavus was the most commonly isolated species. Similarly, Abriba et al (2013) reported that most predominant genera associated with maize and other food grains was Aspergillus. Morphological characteristics are the primary tools in the identification of various Aspergillus species. Despite originating from different districts, isolates showed similarities in their morphological and cultural characteristics. There was not much variation in colony color and pigmentation of A. flavus and A. niger isolates (Reddy et al., 2010; Gautam and Bhadauria, 2012, Odhiambo et al., 2013 and Baquiao et al., 2013). However, there was significant difference among isolates with regard to colony diameter. Isolate of Jaland- har district was fast growing as compared to other districts. Least growth rate was observed in isolates of Ludhiana and Kapurthala district. Several workers (Kumar et al., 2014, Navya et al., 2014 and Amrita and Richa, 2014) have also reported significant differences in growth rate of Aspergillus isolates.

Observations on quantitative characters revealed that twenty isolates of $A$. flavus formed sclerotia and were of the L-strain type having sclerotia diameter of more than $400 \mu \mathrm{m}$. Nayak et al., (2014) and Nyongesa et al., (2015) also reported that A. flavus produed sclerotia of L-strain type that is having sclertioal diameter more than $400 \mu \mathrm{m}$. The size of conidiophores of Aspergillus spp. isolates varied greatly in length and slightly in width. Conidia diameter also shows slight variation in all the isolates. Variations in conidiophore length and conidia size of A. flavus and A. niger was also observed by Reddy et al. (2010).

\section{Conclusion}

The observations of present study showed that $A$. flavus was the most predominantly associated species with maize ear rot in Punjab. Its incidence was found maximum in Hoshiarpur, the major maize growing district of Punjab. The isolates of Jalandhar district were fast growing as compared to Kapurthala district. The present study contributed to the knowledge on diversity of $A$. flavus and A. niger associated with maize ear rot. Sclerotia producing isolates of A. flavus were of L-strain type having sclerotia diameter more 
than $400 \mu \mathrm{m}$. Though the clusters varied from place to place but high level of morphological and cultural diversity existed among the isolates A. flavus and A. niger.

\section{REFERENCES}

Abriba, C., Lennox, J.A., Asikong, B.E, Asitok, A., Ikpoh, I.S., Henshaw, E.E., and Eja, M.E. (2013). Isolation of aflatoxin producing species of Aspergillus from foodstuffs sold in calabar markets, Cross River State, Nigeria. Journal of Microbiology and Biotechnology Research, 3: 8-13.

Amaike, S. and Keller, N.P. (2011). Aspergillus flavus. Annual Review of Phytopathology 49: 107-133.

Amrita, S. and Richa, S. (2014). Biocontrol and environmental studies on paper degrading mycolflora isolated from Sanganer area, Jaipur, India. International Journal of Current Microbiology and Applied Science, 3: 948956.

Anonymous, (2015). HTML color codes and names, retrieved from www.computerhope.com/htmcolor.htm

Atehnkeng, J., Ojiambo, P.S., Donner. M., Ikotun, T., Sikora, R.A., Cotty, P.J. and Bandyopadhyay, R. (2008). Distribution and toxigenicity of Aspergillus species isolated from maize kernels from three agro-ecological zones in Nigeria. International Journal of Food and Microbiology, 122: 74-84.

Baquiao, A.C., Oliveira, M.M.M.D., Reis, T.A., Zorzete, P., Atayde, D.D. and Correa, B. (2013). Polyphasic approach to the identification of Aspergillus sction Flavi isolated from Brazil nuts. Food Chemistry, 139: 1127-1132.

Bello, O.B., Ganiyu, O.T., Wahab, M.K.A., Azeez, M.A., Abdulmaliq, S.Y., Ige, S.A., Mahmood, J., Oluleye, F. and Afolabi, M.S. (2012). Yield and Disease Reactions of Quality Protein Maize Varieties in the Southern Guinea Savanna Agro-Ecology of Nigeria. International Journal of Agriculture and Forestry, 2: 203-209.

Gautam, A.K. and Bhadauria, R. (2012). Characterization of Aspergillus species associated with commercially stored triphala powder. African Journal of Biotechnology, 11: 16814-16823.

Gxasheka, M., Wang, J., Tyasi, T.L. and Gao, J. (2015). Scientific understanding and effects on ear rot diseases in maize production: a review. International Journal of Soil and Crop Sciences, 3: 77-84.

Klich, M.A. (2002). Identification of common Aspergillus Species. CBS Utrecht, Netherlands, Pp. 116.

Kumar, M.R., Sudhakar, P., Santhoshi, M.V.M., Krishna, T.G. and Reddy, K.R. (2014). Cultural, morphological and pathological variability among isolates of Aspergillus flavus in Maize collected from different parts of Andhra Pradesh. Journal of Plant and Pest Science, 1: 9-16.

McClenny, N. (2005). Laboratory detection and identification of Aspergillus species by microscopic observation and culture: the traditional approach. Journal of Medical and Veterinary Mycology, 43: 125-128.

Navya, H.M., Naveen, J., Hariprasad, P. and Niranjana, S.R. (2014). Morphological, physiological and biochemical characterization of Aspergillus flavus isolates from Groundnut (Arachis hypogaea L.). International Journal of Scientific Research, 5: 1777-1783.

Nayak, S., Dhua, U. and Samanta, S. (2014). Occurrence of aflatoxigenic A. flavus in stored rice and rice based products of coastal Odisha, India. International Journal of Current Microbiology and Applied Sciences, 3: 170-181.

Nicholson, P., Gosman, N., Draeger, R. and Steed, A. (2004). Control of Fusarium and Aspergillus species and associated mycotoxins on wheat and maize. In: Meeting the mycotoxins menace, Barug, D., Egmond, H.V., Lopez-Garcia, R., Osenbruggen, T.V. and Visconti, A. (Eds.). Wageningen Academic Publishers, Netherland, pp. 113-126.

Nyongesa, B.W., Okoth, S. and Ayugi, V. (2015). Identification key for Aspergillus species isolated from maize and soil of Nandi County, Kenya. Advances in Microbiology, 5: 205-229.

Odhiambo, B.O., Murage, H. and Wagara, I.N. (2013). Isolation and characterization of aflatoxigenic Aspergillus species from maize and soil samples from selected counties of Kenya. African Journal of Microbiology Research, 7: 4379-488.

Reddy, K.R.N., Farhana, N.I., Wardah, A.R. and Salleh, B. (2010). Morphological identification of foodborne pathogens colonizing Rice grains in South Asia. Pakistan Journal of Biological Sciences, 13: 794-801. 